\title{
Mitral Valve Regurgitation
}

National Cancer Institute

\section{Source}

National Cancer Institute. Mitral Valve Regurgitation. NCI Thesaurus. Code C50852.

The backward flow of blood from the left ventricle into the left atrium, owing to insufficiency of the mitral valve; it may be acute or chronic, usually due to mitral valve prolapse, rheumatic heart disease or a complication of cardiac dilatation. 